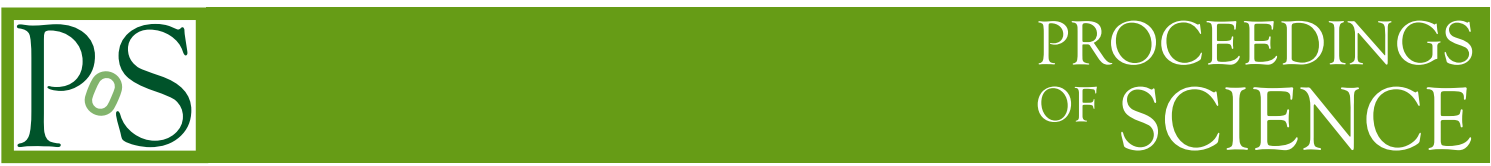

\title{
Soft Supersymmetry Searches at ATLAS and CMS
}

\section{Mateusz Zarucki* on behalf of the ATLAS and CMS Collaborations}

*Institute of High Energy Physics (HEPHY) of the Austrian Academy of Sciences, Vienna, Austria

E-mail: mateusz.zarucki@cern.ch

Soft supersymmetry (SUSY) searches that target models with compressed mass spectra are highly motivated by theoretical considerations, such as naturalness or dark matter relic constraints. This paper gives a concise overview of the most recent results from the ATLAS and CMS Experiments that target soft SUSY signatures, with a focus on the underlying analysis tools and techniques that allow these regions of phase space to be probed. These include sophisticated analysis methods ranging from the identification of soft objects and initial state radiation jets, to specific experimental observables, such as Recursive Jigsaw Reconstruction variables. Improved triggering techniques are also instrumental in expanding the accessible phase space. Different theoretical models are considered, involving strong, electroweakino and vector-boson fusion production, as well as a soft hadronically-decaying tau model. The presented searches are based on proton-proton collisions data from Run II of the Large Hadron Collider, recorded with the ATLAS and CMS detectors at a centre-of-mass energy $\sqrt{s}=13 \mathrm{TeV}$, corresponding to integrated luminosities ranging from $35.9 \mathrm{fb}^{-1}$ to $139 \mathrm{fb}^{-1}$.

The Eighth Annual Conference on Large Hadron Collider Physics-LHCP2020

25-30 May, 2020

online 


\section{Introduction}

In the absence of recent discoveries at the Large Hadron Collider (LHC) [1] and the exclusion of wide regions [2,3] on a number of SUSY [4] models, there is significant pressure on classical types of searches for new physics, which are more inclusive in their methodology. Soft SUSY searches are more targeted analyses that consider models with compressed mass spectra, where the mass difference $\Delta m$ between the lightest supersymmetric particle (LSP) and next-to-LSP (NLSP) is relatively small. The possible decay modes and kinematics are dictated by the level of compression of $\Delta m$. There are additional theoretical motivations for SUSY models with compressed mass spectra, such as the prediction that co-annihilation processes of LSPs could reproduce the correct cosmological dark matter relic density [5,6]. Furthermore, they can still provide a window to natural SUSY [7-9], as the searches typically focus on lower mass scales, which have not yet been excluded due to the lower cross sections and difficulty of the analyses. There is a significant effort at the ATLAS [10] and CMS [11] Experiments focusing on soft SUSY. This paper provides an overview of the underlying analysis techniques of the most recent results targeting these signatures.

\section{Signature and Models}

High $\Delta m$ compression results in little available energy and therefore 'soft' visible decay products, which have low transverse momenta $p_{\mathrm{T}}$. These products, including the missing transverse momentum $p_{\mathrm{T}}^{\text {miss }}$, typically would not pass the detector acceptance thresholds. In the presence of initial state radiation (ISR), the system is boosted and the visible decay products and $p_{\mathrm{T}}^{\text {miss }}$ signatures become detectable. In the case of vector-boson fusion (VBF) topologies, the standard signature includes two high $p_{\mathrm{T}}$ forwards jets. Therefore, a typical soft SUSY signature comprises at least one high transverse momentum jet ( $p_{\mathrm{T}} \gtrsim 100 \mathrm{GeV}$ ), moderate $p_{\mathrm{T}}^{\text {miss }}$ and soft leptons or jets.

The results are interpreted over a wide range of different simplified models [12,13], depending on the production mechanisms and decay modes that are governed by $\Delta m$. Strong production focuses on the stop $\tilde{t}$ pair-production with a neutralino LSP $\tilde{\chi}_{1}^{0}$ and the final states are classified according to the $\mathrm{W}$ boson decay modes [14]. In the case of very compressed scenarios where $\Delta m<m_{\mathrm{W}}$, the decay proceeds via an off-shell top $\mathrm{t}^{*}$ and $\mathrm{W}^{*}$, resulting in a four-body final state [15-18]. The same final state is possible with a decay via an intermediate chargino $\tilde{\chi}_{1}^{ \pm}$, if its mass is lower than the stop mass [15, 19]. A possible competing process involves the decay to a charm quark, via a flavour-changing neutral current (FCNC) interaction $[16,20]$.

Soft SUSY searches are also sensitive to compressed electroweak (EWK) models. Neutralinos $\tilde{\chi}_{1}^{0}$ and charginos $\tilde{\chi}_{1}^{ \pm}$are collectively referred to as electroweakinos (EWKinos) and depending on the mass parameters of the MSSM [21, 22], they could have have a wino, bino or higgsino-like nature. Many analyses consider chargino-neutralino $\left(\tilde{\chi}_{1}^{ \pm}-\chi_{2}^{0}\right)$ production where the compressed region is interpreted in terms of an off-shell $\mathrm{W}^{*} \mathrm{Z}^{*}$-mediated model $[19,23,24]$. Results are also interpreted in terms of the phenomenological MSSM (pMSSM) [19, 25]. Leptonic searches also include the scenario of direct pair-production of sleptons $\tilde{\ell}$ [23].

The VBF production of EWKinos and their decays via off-shell $\mathrm{W}^{*} \mathrm{Z}^{*}, \mathrm{~W}^{*} \mathrm{~W}^{*}$, or intermediate sleptons $\tilde{\ell}$, result in final states with two jets and two soft leptons [23, 26]. A first interpretation of direct and indirect (via EWKinos) production of stau $\tilde{\tau_{h}}$ particles with a soft hadronically-decaying tau $\tau$ has been performed, targeting $\Delta m$ between the $\tilde{\chi}_{1}^{0}$ and $\tilde{\tau}_{h}$ of $\approx 50 \mathrm{GeV}$ [27]. 


\section{Analysis Methods}

\subsection{Soft Object Identification}

The ability for the detectors to efficiently reconstruct soft objects is crucial in the context of compressed SUSY signatures. The experiments are able to reconstruct muons down to $3 \mathrm{GeV}$ [2830] and electrons down to $4.5 \mathrm{GeV}$ [31,32]. Some searches [26] include signal regions targeting $1<\Delta m<10 \mathrm{GeV}$, where multiple signal leptons are not expected to be identified due to their extremely low $p_{\mathrm{T}}$. The compressed EWKino search [23] uses a signal region based on a lepton and an opposite-sign isolated low- $p_{\mathrm{T}}$ track to increase the sensitivity for the lowest $\Delta m$. For these regions, the track is selected to be matched to a reconstructed lepton candidate with no identification requirements, allowing electron and muon candidates to be reconstructed with $p_{\mathrm{T}} \mathrm{s}$ as low as $1 \mathrm{GeV}$ and $2 \mathrm{GeV}$, respectively. The analysis also identifies muons using a LowPt ID criterion [29], which has a selection efficiency of $\approx 90 \%$ for $p_{\mathrm{T}}<5 \mathrm{GeV}$ (Figure 1 left). In some searches [26, 27], soft hadronically-decaying taus $\tau_{h}$ are also reconstructed with $20<p_{\mathrm{T}}<40 \mathrm{GeV}$ as signal.

Standard b-tagging algorithms of heavy-flavour jets rely on the reconstruction of displaced secondary vertices due to the finite lifetimes of b-hadrons [33, 34]. Many compressed analyses with top quark decays in the signal veto b-tagged jets [15, 19, 23, 24, 26, 27], as they are expected to be too soft for reconstruction by standard algorithms that rely on calorimeter jet reconstruction $\left(p_{\mathrm{T}}>20 \mathrm{GeV}\right)$. Several analyses [16-18, 35] take advantage of the sophisticated soft b-tagging algorithms [36, 37], which allow b-tagged jets to be identified with $1<p_{\mathrm{T}}<20 \mathrm{GeV}$ (Figure 1 right), which is important for the smallest $\Delta m$. The CMS Inclusive Vertex Finder (IVF) [37] and the ATLAS Track-based Low- $p_{\mathrm{T}}$ Vertex Tagger (T-LVT) [36] algorithms exploit the excellent tracking capabilities of the detectors in order to reconstruct soft b-tagged jets only from tracks or from secondary vertices without the strict requirement of association to a calorimeter jet.
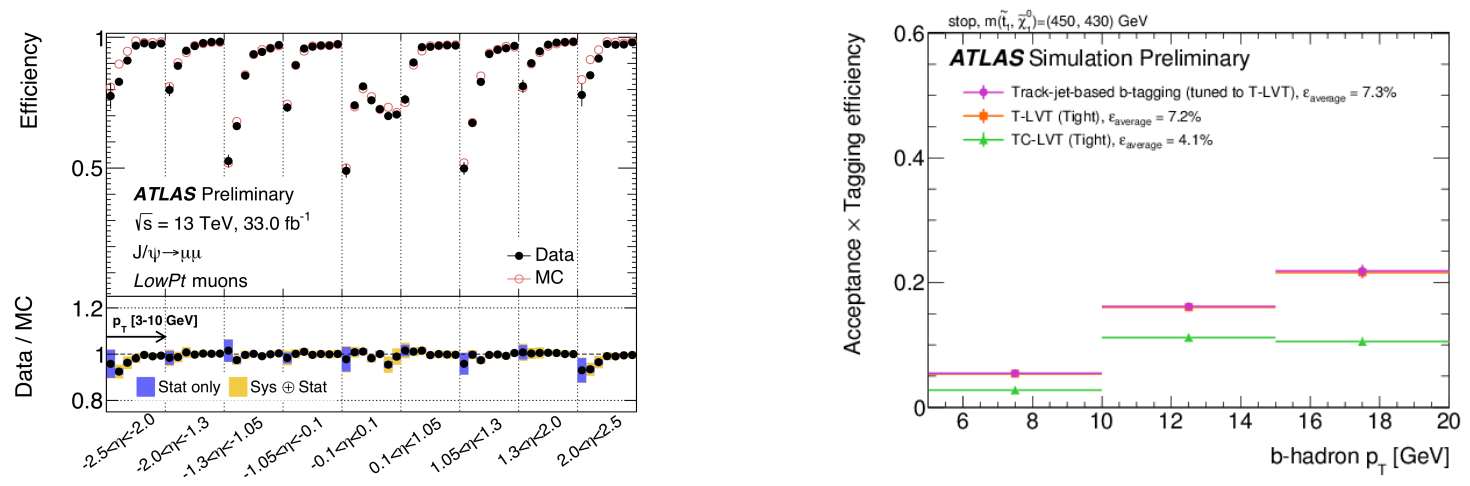

Figure 1: Efficiency of LowPt ID in data (left) [29] and soft b-tagging algorithms in simulation (right) [36]

\subsection{Initial State Radiation}

The characteristic signature of compressed searches is the presence of a high momentum ISR jet originating from the hard scattering process of interacting partons. ISR jets are predicted to predominantly originate from gluons, and due to the varying QCD interactions and subsequent hadronisation, they exhibit different properties depending on the flavour of the original parton. Gluon-initiated jets have a higher particle multiplicity, a more uniform fragmentation function and 
are less collimated than quark jets $[38,39]$. Therefore, gluon ISR jets can be tagged in order to distinguish them from certain backgrounds or signal quark jets. An example involves clustering jets with a larger sized cone, yielding large- $R$ jets [16]. The Quark-Gluon Likelihood discriminant $[38,40]$ is a tool which uses the jet properties in order to distinguish gluon from light quark jets.

In SUSY models, the signature left by LSPs in the form of missing transverse momentum $p_{\mathrm{T}}^{\text {miss }}$ is often indistinguishable from SM backgrounds such as neutrinos. The recoil of the LSPs against a high $p_{\mathrm{T}}$ ISR jet can provide additional indirect sensitivity and separation from the backgrounds. The Recursive Jigsaw Reconstruction (RJR) [41, 42] is a technique that allows recursively imposing a given decay tree on reconstructed events, to yield observables that can exploit kinematic correlations. In the case of compressed ISR signatures, RJR sub-divides the topology into an ISR system, as well as visible and invisible sparticle systems, yielding an estimator for the ISR recoil $\mathrm{R}_{\mathrm{ISR}}=I S R-p_{\mathrm{T}} / p_{\mathrm{T}}^{\text {miss }}$ $[18,23]$. Since the calculation resolves potential kinematic and combinatoric ambiguities, such as the provenance of jets, this quantity is more accurate in events with multiple radiated jets. In the case of VBF topologies, a similar discriminating variable $R_{V B F}$ can be constructed by replacing the ISR system in $R_{I S R}$ by the vector sum of the two VBF jets [23].

\subsection{Triggers}

A number of soft SUSY searches rely on an online trigger $[10,11]$ selection on pure $p_{\mathrm{T}}^{\text {miss }}$ [15-18, 23, 26, 27], which plateaued at $p_{\mathrm{T}}^{\text {miss }} \approx 250 \mathrm{GeV}$. Lowering the $p_{\mathrm{T}}^{\text {miss }}$ trigger thresholds would increase the acceptance for compressed signatures, which have moderate $p_{\mathrm{T}}^{\text {miss }}$. Moreover, it would provide a window to less accessible models with lower cross sections [43] or difficult phase spaces, such as compressed EWKinos or higgsinos. One of the main improvements of the soft dilepton analysis [19] was the development of a dedicated dimuon $\left(p_{\mathrm{T}}>3 \mathrm{GeV}\right)$ and $p_{\mathrm{T}}^{\text {miss }}$ crosstrigger, in order to expand the phase space to a lower $p_{\mathrm{T}}^{\text {miss }}$ signal region, increasing the efficiency and sensitivity of the analysis. Furthermore, finding ways to reduce the rates of the $p_{\mathrm{T}}^{\text {miss }}$ triggers is crucial in mitigating the increase in trigger thresholds due to the higher instantaneous luminosity and harsher pileup conditions at the LHC. A soft $\left(p_{\mathrm{T}}>3 \mathrm{GeV}\right)$ muon, high $p_{\mathrm{T}}>100 \mathrm{GeV}$ jet and $p_{\mathrm{T}}^{\text {miss }}$ cross-trigger has been developed, exploiting the typical ISR signature, in order to achieve lower $p_{\mathrm{T}}^{\text {miss }}$ thresholds by $\approx 50 \mathrm{GeV}[15,44]$. An algorithm triggering on the VBF topology with a soft muon $\left(p_{\mathrm{T}}>4 \mathrm{GeV}\right)$ has been developed to reduce the $p_{\mathrm{T}}^{\text {miss }}$ thresholds $[26,45]$.

\section{Conclusion and Outlook}

There is a significant effort at the ATLAS and CMS Experiments covering the compressed SUSY phase space. Soft SUSY signatures can be probed with the help of a number of analysis techniques, ranging from identification of soft objects and ISR to sophisticated experimental observables. Many innovative methods are being developed and used, such as improved triggering techniques for expanding the accessible phase space, or the use of machine learning algorithms for object identification or signal region definitions [15]. In terms of theory, there is the possibility of extending the existing models to displaced signatures for the smallest $\Delta m$ [14]. An increased integrated luminosity is not expected to bring large gains in sensitivity for many searches [46]. Therefore, we need to take advantage of these techniques and go beyond initial design considerations of the experiments to really push to the limits of detection, analysis and theory. 


\section{References}

[1] L. Evans and P. Bryant, LHC Machine, Journal of Instrumentation 3 (2008) S08001.

[2] ATLAS Collaboration, SUSY July 2020 Summary Plot Update, ATL-PHYS-PUB-2020-020. https://cds.cern.ch/record/2725258.

[3] CMS Collaboration, Run 2 Summary Plots (13 TeV), Twiki. https://twiki.cern.ch/twiki/bin/view/CMSPublic/PhysicsResultsSUS.

[4] S. P. Martin, A Supersymmetry Primer, Advanced Series on Directions in High Energy Physics 18 (1998) 1, [hep-ph/9709356].

[5] C. Balázs, M. Carena and C. E. M. Wagner, Dark Matter, Light Stops and Electroweak Baryogenesis, Physical Review D 70 (2004) 015007, [hep-ph/0403224].

[6] J. Edsjö and P. Gondolo, Neutralino Relic Density including Coannihilations, Physical Review D 56 (1997) 1879-1894, [hep-ph/9704361].

[7] R. Barbieri and G. Giudice, Upper Bounds on Supersymmetric Particle Masses, Nuclear Physics B 306 (1987) 63-76.

[8] G. F. Giudice, T. Han, K. Wang and L.-T. Wang, Nearly Degenerate Gauginos and Dark Matter at the LHC, Physical Review D 81 (2010) 115011, [1004 . 4902].

[9] A. Mustafayev and X. Tata, Supersymmetry, Naturalness, and Light Higgsinos, Indian Journal of Physics 88 (2014) 991-1004, [1404 . 1386].

[10] ATLAS Collaboration, The ATLAS Experiment at the CERN Large Hadron Collider, Journal of Instrumentation 3 (2008) S08003.

[11] CMS Collaboration, The CMS Experiment at the CERN LHC, Journal of Instrumentation 3 (2008) S08004.

[12] J. Alwall, P. Schuster and N. Toro, Simplified Models for a First Characterization of New Physics at the LHC, Physical Review D 79 (2009) 075020, [0810 . 3921].

[13] LHC New Physics Working Group Collaboration, Simplified Models for LHC New Physics Searches, Journal of Physics G 39 (2012) 105005, [1105 . 2838].

[14] R. Gröber, M. M. Mühlleitner, E. Popenda and A. Wlotzka, Light Stop Decays: Implications for LHC Searches, The European Physical Journal C 75 (2015) 420, [1408 . 4662].

[15] CMS Collaboration, Search for top squarks decaying via four-body or chargino-mediated modes in single-lepton final states in proton-proton collisions at $\sqrt{s}=13 \mathrm{TeV}$, Journal of High Energy Physics 09 (2018) 065, [1805 . 05784].

[16] CMS Collaboration, Search for direct production of supersymmetric partners of the top quark in the all-jets final state in proton-proton collisions at $\sqrt{s}=13 \mathrm{TeV}$, Journal of High Energy Physics 10 (2017) 005, [1707. 03316]. 
[17] ATLAS Collaboration, Search for new phenomena with top quark pairs in final states with one lepton, jets, and missing transverse momentum in pp collisions at $\sqrt{s}=13 \mathrm{TeV}$ with the ATLAS detector, ATLAS-CONF-2020-003. https://cds.cern.ch/record/2711489.

[18] ATLAS Collaboration, Search for a scalar partner of the top quark in the all-hadronic tit plus missing transverse momentum final state at $\sqrt{s}=13 \mathrm{TeV}$ with the ATLAS detector, The European Physical Journal C 80 (2020) 737, [2004.14060].

[19] CMS Collaboration, Search for new physics in events with two soft oppositely charged leptons and missing transverse momentum in proton-proton collisions at $\sqrt{s}=13 \mathrm{TeV}$, Physics Letters B 782 (2018) 440 - 467, [1801.01846].

[20] CMS Collaboration, Search for the pair production of third-generation squarks with two-body decays to a bottom or charm quark and a neutralino in proton-proton collisions at $\sqrt{s}=13 \mathrm{TeV}$, Physics Letters B 778 (2018) 263 - 291, [1707. 07274].

[21] P. Fayet, Supersymmetry and Weak, Electromagnetic and Strong Interactions, Physics Letters B 64 (1976) 159.

[22] P. Fayet, Spontaneously Broken Supersymmetric Theories of Weak, Electromagnetic and Strong Interactions, Physics Letters B 69 (1977) 489.

[23] ATLAS Collaboration, Searches for electroweak production of supersymmetric particles with compressed mass spectra in $\sqrt{s}=13 \mathrm{TeV}$ pp collisions with the ATLAS detector, Physical Review D 101 (2020) 052005, [1911.12606].

[24] ATLAS Collaboration, Search for chargino-neutralino pair production in final states with three leptons and missing transverse momentum in $\sqrt{s}=13 \mathrm{TeV} p-p$ collisions with the ATLAS detector, ATLAS-CONF-2020-015. https://cds.cern.ch/record/2719521.

[25] MSSM Working Group Collaboration, The Minimal Supersymmetric Standard Model: Group Summary Report, in GDR (Groupement de Recherche) - Supersymétrie, 1998, hep-ph/9901246, https://cds.cern.ch/record/376049.

[26] CMS Collaboration, Search for supersymmetry with a compressed mass spectrum in the vector boson fusion topology with 1-lepton and 0-lepton final states in proton-proton collisions at $\sqrt{s}=13 \mathrm{TeV}$, Journal of High Energy Physics 08 (2019) 150, [1905 . 13059].

[27] CMS Collaboration, Search for supersymmetry with a compressed mass spectrum in events with a soft $\tau$ lepton, a highly energetic jet, and large missing transverse momentum in proton-proton collisions at $\sqrt{s}=13 \mathrm{TeV}$, Physical Review Letters 124 (2020) 041803, [1910.01185].

[28] ATLAS Collaboration, Muon reconstruction performance of the ATLAS detector in proton-proton collision data at $\sqrt{s}=13 \mathrm{TeV}$, The European Physical Journal C 76 (2016) 292, [1603.05598]. 
[29] ATLAS Collaboration, Identification of very-low transverse momentum muons in the ATLAS experiment, ATL-PHYS-PUB-2020-002. https://cds.cern.ch/record/2710574.

[30] CMS Collaboration, Muon Reconstruction and Identification Performance with Run-2 Data, CMS-DP-2020-040. https://cds.cern.ch/record/2727091.

[31] ATLAS Collaboration, Electron reconstruction and identification in the ATLAS experiment using the 2015 and 2016 LHC proton-proton collision data at $\sqrt{s}=13 \mathrm{TeV}$, The European Physical Journal C 79 (2019) 639, [1902 . 04655].

[32] CMS Collaboration, Performance of electron reconstruction and selection with the CMS detector in proton-proton collisions at $\sqrt{s}=8 \mathrm{TeV}$, Journal of Instrumentation 10 (2015) P06005, [1502. 02701].

[33] ATLAS Collaboration, ATLAS b-jet identification performance and efficiency measurement with $\mathrm{t} \overline{\mathrm{t}}$ events in pp collisions at $\sqrt{s}=13 \mathrm{TeV}$, The European Physical Journal C 79 (2019) 970, [1907.05120].

[34] CMS Collaboration, Identification of heavy-flavour jets with the CMS detector in pp collisions at $13 \mathrm{TeV}$, Journal of Instrumentation 13 (2018) P05011, [1712.07158].

[35] CMS Collaboration, Search for direct top squark pair production in events with one lepton, jets, and missing transverse momentum at $13 \mathrm{TeV}$ with the CMS experiment, Journal of High Energy Physics 05 (2020) 032, [1912 . 08887].

[36] ATLAS Collaboration, Soft b-hadron tagging for compressed SUSY scenarios, ATLAS-CONF-2019-027. https://cds.cern.ch/record/2682131.

[37] CMS Collaboration, Measurement of $B \bar{B}$ angular correlations based on secondary vertex reconstruction at $\sqrt{s}=7 \mathrm{TeV}$, Journal of High Energy Physics 03 (2011) 136, [1102 . 3194].

[38] CMS Collaboration, Performance of quark/gluon discrimination in $8 \mathrm{TeV}$ pp data, CMS-PAS-JME-13-002. https://cds.cern.ch/record/1599732.

[39] ATLAS Collaboration, Discrimination of Light Quark and Gluon Jets in pp collisions at $\sqrt{s}=8 \mathrm{TeV}$ with the ATLAS Detector, ATLAS-CONF-2016-034. https://cds.cern.ch/record/2200202.

[40] CMS Collaboration, Performance of quark/gluon discrimination in $13 \mathrm{TeV} \mathrm{data,}$ CMS-DP-2016-070. https://cds.cern.ch/record/2234117.

[41] P. Jackson and C. Rogan, Recursive Jigsaw Reconstruction: HEP event analysis in the presence of kinematic and combinatoric ambiguities, Physical Review D 96 (2017) 112007, [1705. 10733].

[42] P. Jackson, C. Rogan and M. Santoni, Sparticles in Motion: Analyzing compressed SUSY scenarios with a new method of event reconstruction, Physical Review D 95 (2017) 035031, [1607.08307]. 
[43] M. Kramer, A. Kulesza, R. van der Leeuw, M. Mangano, S. Padhi, T. Plehn et al., Supersymmetry production cross sections in pp collisions at $\sqrt{s}=7 \mathrm{TeV},[1206.2892]$.

[44] CMS Collaboration, Performance of a Soft Muon, Hard Jet and Moderate Missing Energy Trigger in 2018 Data, CMS-DP-2020-004. https://cds.cern.ch/record/2710122.

[45] CMS Collaboration, SUSY Mu+VBF Trigger Performance with 2017 Data, CMS-DP-2020-014. https://cds.cern.ch/record/2714881.

[46] CMS Collaboration, Searches for light higgsino-like charginos and neutralinos at the HL-LHC with the Phase-2 CMS detector, CMS-PAS-FTR-18-001. https://cds.cern.ch/record/2648538. 